\title{
Hydrogen peroxide-producing pyruvate oxidase from Lactobacillus delbrueckii is catalytically activated by phosphotidylethanolamine
}

\author{
Louis P. Cornacchione* and Linden T. Hu
}

\begin{abstract}
Background: Pyruvate oxidase (Pox) is an important enzyme in bacterial metabolism for increasing ATP production and providing a fitness advantage via hydrogen peroxide production. However, few Pox enzymes have been characterized from bacterial species. The tetrameric non-hydrogen-peroxide producing Pox from E. coli is activated by phospholipids, which is important for its function in vivo.

Results: We characterized the hydrogenperoxide-producing Pox from L. delbrueckii strain STYM1 and showed it is specifically activated by phosphotidylethanolamine (16:0-18:1), but not by phosphotidylcholine or phosphotidylglycerol. This activation is a mixture of $\mathrm{K}$ - and $\mathrm{V}$-type activation as both $\mathrm{k}_{\mathrm{m}}$ and enzyme turnover are altered. Furthermore, we demonstrated that the L. delbrueckii Pox forms pentamers and either decamers or dimers of pentamers in solution, which is different from other characterized Pox enzymes. Lastly, we generated a Cterminal truncation mutant that was only weakly activated by phosphotidylethanolamine, which suggests the Cterminus is important for lipid activation.

Conclusions: To our knowledge this is the first known hydrogenperoxide-producing Pox enzyme that is activated by phospholipids. Our results suggest that there are substantial differences between Pox enzymes from different bacterial species, which could be important for their role in biological systems as well as in the development of Pox-based biosensors.
\end{abstract}

Keywords: Catalytic activation, Hydrogen peroxide, Lactobacillus delbrueckii, Pyruvate oxidase, Phospholipids

\section{Background}

Pyruvate oxidase (Pox) is an enzyme in the oxidoreductase family. There are hydrogen peroxide-producing and acetate-producing Pox enzymes. In hydrogenperoxideproducing Pox enzymes, pyruvate, phosphate, and oxygen are converted to acetylphosphate, carbon dioxide, and hydrogen peroxide with the cofactors flavin adenine dinucleotide (FAD) and thiamine pyrophosphate (TPP) [1].

\footnotetext{
* Correspondence: Lou.Cornacchione@gmail.com Department of Molecular Biology and Microbiology, Tufts University School of Medicine, Boston, MA 02111, USA
}

Acetate-producing Pox enzymes catalyze the oxidative decarboxylation of pyruvate to form carbon dioxide and acetate with electrons being transferred directly to the electron transport chain via the membrane-embedded electron carriers $[2,3]$.

The most well studied acetate-producing Pox is from Escherichia coli. E. coli Pox is dependent on the same cofactors as other Pox enzymes and is also homo-tetrameric [4]. E. coli Pox produces acetate and does not utilize oxygen as its final electron acceptor, but rather the membrane embedded ubiquinone 8 electron transport molecule [2].

(c) The Author(s). 2020 Open Access This article is licensed under a Creative Commons Attribution 4.0 International License, which permits use, sharing, adaptation, distribution and reproduction in any medium or format, as long as you give appropriate credit to the original author(s) and the source, provide a link to the Creative Commons licence, and indicate if changes were made. The images or other third party material in this article are included in the article's Creative Commons licence, unless indicated otherwise in a credit line to the material. If material is not included in the article's Creative Commons licence and your intended use is not permitted by statutory regulation or exceeds the permitted use, you will need to obtain permission directly from the copyright holder. To view a copy of this licence, visit http://creativecommons.org/licenses/by/4.0/. The Creative Commons Public Domain Dedication waiver (http://creativecommons.org/publicdomain/zero/1.0/) applies to the data made available in this article, unless otherwise stated in a credit line to the data. 
CidC is another acetate-producing Pox enzyme from Staphylococcus aureus which transfers electrons to menaquinone and has an important role in cell death pathways [3]. Pyruvate:Quinone Oxidoreductase (PQO) from Corynebacterium glutamicum also produces acetate and transfers electrons to a quinone [5]. One characteristic of E. coli Pox, PQO, and CidC is that they are catalytically activated by phospholipids, which is thought to facilitate efficient transfer to membrane electron shuttles by activating the enzyme at a membrane peripheral position $[3,5$, 6]. Mutants in E. coli Pox that disrupt lipid activation of the enzyme are localized to the $\mathrm{C}$-terminal region of the protein. Further biochemical analysis identified a lipid activation helix in the C-terminal region (amino acids 558568) that upon lipid interaction induce the preceding alpha helix to move out of the active site, which in turn positions phenylalanine 465 into the active site enhancing electron transfer between TPP and FAD [7-9]. The phospholipid activation of E. coli Pox is a hybrid of $\mathrm{K}_{\mathrm{m}}$ driven (K-type) and velocity-driven (V-type) allosteric activation where the $\mathrm{k}_{\mathrm{m}}$ for pyruvate decreases along with an increase in the enzyme turnover rate [10]. However, in CidC the activation is V-type where the enzyme turnover rate is substantially increased [3].

Hydrogen peroxide-generating Pox enzymes are produced by multiple different bacteria. Pox enzymes from Lactobacillus plantarum and Streptococcus pneumoniae are the most well studied. These enzymes form homotetrameric structures $[11,12]$. The role of Pox enzymes in central metabolism is thought to be increasing ATP production in concert with acetate kinase during aerobic metabolism [13]. In S. pneumoniae, which has no tricarboxylic acid (TCA) cycle, additional ATP production from glucose is believed to come from this pathway [10]. L. plantarum Pox is considered to be an important component of the enhanced biomass that is observed in aerobic growth by generating acetyl phosphate as a substrate for acetate kinase [13]. In addition, the production of hydrogen peroxide from Pox could also be important on a community level. Since hydrogen peroxide is toxic to some bacteria, Pox activity could confer a fitness advantage to the producer organism. In S. pneumoniae, pox-deficient mutants display decreased virulence in a rat model of disease likely through decreased adhesion properties, but also potentially through decreased competition with commensal bacteria [14]. Streptococcus gordonii and Streptococcus sanguinis are known to produce enough hydrogen peroxide primarily through Pox to inhibit the oral pathogen Streptococcus mutans [15]. Recently, Lactobacillus delbrueckii strains harboring intact pox genes were shown to produce enough hydrogen peroxide to inhibit the growth of the oral pathogen Porphyromonas gingivalis [16]. The characterization of hydrogen peroxide-producing strains and their respective encoded hydrogen peroxide-producing enzymes could be beneficial in the development of novel treatments for diseases like periodontitis.

In the current study, we characterize a hydrogen peroxideproducing Pox enzyme from $L$. delbrueckii that is catalytically activated specifically by phosphotidylethanolamine, a common bacterial membrane component. This activation is largely dependent on the C-terminal region of the protein. Further, we showed that the L. delbrueckii Pox adopts a pentameric structure and potentially a dimer of pentamers, which is novel for Pox enzymes. These characteristics are unique for hydrogenperoxide-producing Pox enzymes and demonstrate substantial variability in the structure and function of Pox from different bacterial species.

\section{Results}

\section{Characterization of $\mathrm{L}$. delbrueckii pox}

The E. coli Pox has been well studied, and it is known that this enzyme is catalytically activated by phospholipids $[6,7,17]$. In addition, CidC and PQO from $S$. aureus and C. gluticaticum, respectively, are other pyruvate oxidases that are lipid activated $[3,5]$. Another well studied Pox enzyme is from Lactobacillus plantarum $[1,13,18]$. This enzyme has not been shown to be catalytically activated by phospholipids indicating that there is variability in the catalytic activation of the Pox enzymes. Amino acid identity between the enzymes ranges from $25.9 \%$ between L. plantarum Pox and PQO to $46.4 \%$ between E. coli Pox and PQO (Table 1). For DNA sequence identity, the identity ranges from $50.7 \%$ between CidC and PQO to $56.3 \%$ for CidC and L. plantarum Pox (Table 1).

First, we wanted to determine the biochemical properties of the L. delbrueckii Pox enzyme. We previously purified L. delbrueckii Pox and used it for subsequent testing [16]. We determined the $\mathrm{k}_{\mathrm{m}}$ values for pyruvate

Table 1 Amino acid and DNA percent identity of pyruvate oxidase enzymes. Shown is the amino acid and DNA sequence identity of pyruvate oxidase genes and proteins

\begin{tabular}{|c|c|c|c|c|}
\hline \multicolumn{5}{|c|}{ Amino Acid Sequence Identity } \\
\hline Enzyme & E. coli Pox & CidC & PQO & L. plantarum Pox \\
\hline L. delbrueckii Pox & $27.3 \%$ & $33.1 \%$ & $25.8 \%$ & $37.8 \%$ \\
\hline E. coli Pox & & $31.7 \%$ & $46.4 \%$ & $28.8 \%$ \\
\hline CidC & & & $31.3 \%$ & $32.9 \%$ \\
\hline PQO & & & & $25.9 \%$ \\
\hline \multicolumn{5}{|c|}{ DNA Sequence Identity } \\
\hline Enzyme & E. coli Pox & CidC & PQO & L. plantarum Pox \\
\hline L. delbrueckii Pox & $52.4 \%$ & $53.8 \%$ & $52.2 \%$ & $55.7 \%$ \\
\hline E. coli Pox & & $51.3 \%$ & $55.7 \%$ & $52.2 \%$ \\
\hline CidC & & & $50.7 \%$ & $56.3 \%$ \\
\hline PQO & & & & $52.2 \%$ \\
\hline
\end{tabular}


and phosphate and the $\mathrm{k}_{\mathrm{cat}}$ value. The $\mathrm{k}_{\mathrm{m}}$ for pyruvate and phosphate were $342.2 \mu \mathrm{M}$ and $8 \mathrm{mM}$ respectively, and the $\mathrm{k}_{\text {cat }}$ was $0.367 \mathrm{~s}^{-1}$ (Fig. 1 and Table 2). The optimum $\mathrm{pH}$ for several other pyruvate oxidase enzymes is near 5.5 and so we tested the activity of the $L$. delbrueckii Pox at various $\mathrm{pHs}$ to determine its $\mathrm{pH}$ activity profile. In agreement with other Pox enzymes, the $L$. delbrueckii Pox was most active at the $\mathrm{pH}$ range of 5.5 to 6 (Fig. 1c).

\section{L. delbrueckii pox forms pentameric structure in solution}

Both the L. plantarum and E. coli Pox are known to form tetrameric structures $[1,4]$. To determine whether the $L$. delbrueckii Pox also formed a tetramer, we performed gel filtration chromatography with the purified enzyme. Interestingly, L. delbrueckii Pox eluted in two major peaks; the first with a calculated molecular weight of $694 \mathrm{kDa}$ and the second with a calculated molecular weight of $345 \mathrm{kDa}$ (Fig. 2a and b). The molecular weight of an STYM1 L. delbrueckii Pox monomer is $68 \mathrm{kDa}$ according to the translation of the gene sequence; therefore, the first peak corresponded to a decamer and the second peak to a pentamer. The last peak corresponded to unbound FAD (Fig. 2a). Analysis of the fractions by non-reducing SDS-PAGE revealed two distinct bands that migrated distances consistent with monomer and dimer forms of the $L$. delbrueckii Pox at approximately $68 \mathrm{kDa}$ and $136 \mathrm{kDa}$ (Fig. 2c). Fractions 13 and 14, which correspond to the last peak from gel filtration, did not contain any protein and also were yellow in color confirming the presence of unbound FAD (Fig. 2c). To confirm that the $136 \mathrm{kDa}$ protein band was in fact a dimer of $L$. delbrueckii Pox, we analyzed the $L$. delbrueckii Pox on non-reducing and a reducing SDS-PAGE. Under reducing conditions, $L$. delbrueckii Pox migrated exclusively as a monomer indicating that the $136 \mathrm{kDa}$ band is a dimer, presumably linked by a disulfide bond at
Table 2 Kinetic parameters of L. delbrueckii Pox. Values for each parameter are listed with standard error

\begin{tabular}{lll}
\hline Km Pyruvate & Km PO4 & Kcat \\
\hline $342.2 \mu \mathrm{M} \pm 44.9$ & $8 \mathrm{mM} \pm 1.35$ & $0.367 \mathrm{~s}-1 \pm 0.013$ \\
\hline
\end{tabular}

position 72 since it is the only cysteine in the amino acid sequence (Fig. 2d).

\section{Phospholipid activation of $\mathrm{L}$. delbrueckii pox}

We hypothesized that $L$. delbrueckii Pox may be activated by phospholipids similar to $E$. coli Pox, CidC, and PQO $[2,3,5]$. We tested whether addition of either phosphotidylethanolamine (PE), phosphotidylcholine (PC), and phosphotidylglycerol (PG) could catalytically activate L. delbrueckii Pox. L. delbrueckii Pox was catalytically activated specifically by PE, but not PC or PG (Fig. 3a). The phospholipids used contained the same acyl chain structure (16:0-18:1) and only varied in the headgroup indicating that there is specificity in phospholipid activation of $L$. delbrueckii. To determine the full activation profile of $L$. delbrueckii Pox by PE, we measured the fold activity over a wider range of PE concentrations and observed activation upwards of 6 -fold relative to the enzyme without PE (Fig. 3b). This level of activation exceeds that of the CidC Pox (3-fold activation) from S. aureus [3].

To gain mechanistic insight into how PE activates the enzyme, we tested the kinetic parameters of $L$. delbrueckii Pox in the presence of PE (Fig. 4). With the addition of $\mathrm{PE}$, the $\mathrm{k}_{\mathrm{m}}$ for pyruvate and phosphate were $519.9 \mu \mathrm{M}$ and $1.25 \mathrm{mM}$ respectively and the $\mathrm{k}_{\text {cat }}$ was $6.85 \mathrm{~s}^{-1}$ (Table 3). The approximately 18 -fold increase in $\mathrm{k}_{\mathrm{cat}}$ and decrease in phosphate $\mathrm{k}_{\mathrm{m}}$ relative to the enzyme in the absence of PE suggest that these parameters are the primary drivers of catalytic activation, which is
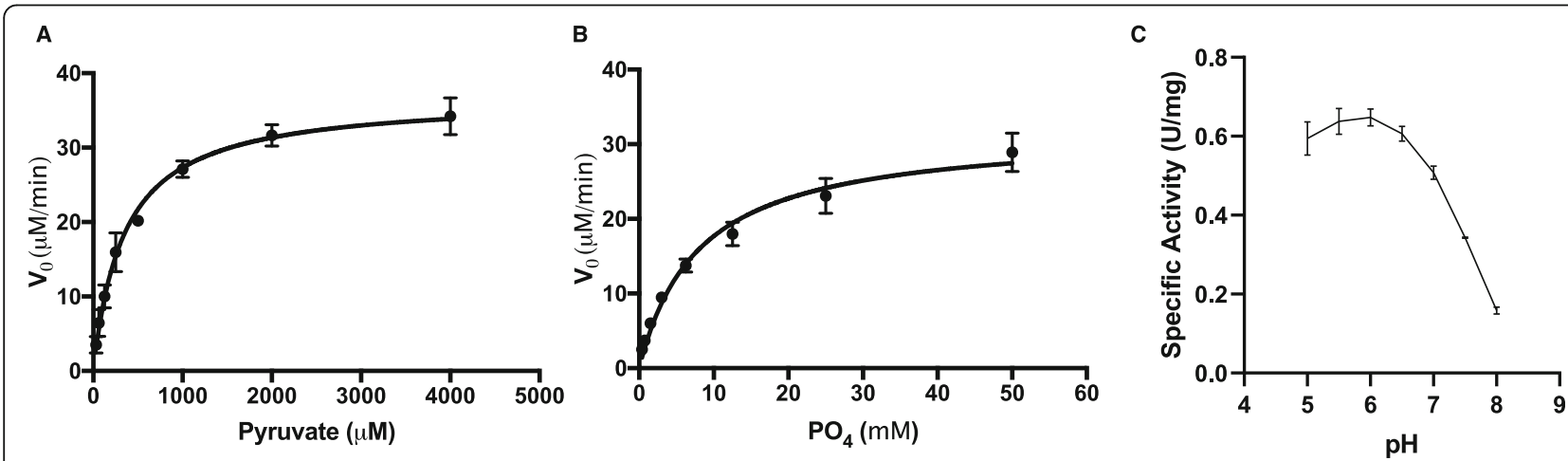

Fig. 1 Kinetic parameters of $L$. delbrueckii Pox. a. Shown is the enzyme velocity of $L$. delbrueckii Pox at various concentrations of pyruvate in the reaction mixture. $\mathbf{b}$. Shown is the enzyme velocity of $L$. delbrueckii Pox at various concentrations of phosphate in the reaction mixture. In both panels, enzyme concentration was $1.76 \mathrm{uM}$. The data represent the average of two independent experiments and error bars represent standard error. c. Shown is the enzyme specific activity at various pHs. The data represent the average of two independent experiments and error bars represent standard error 

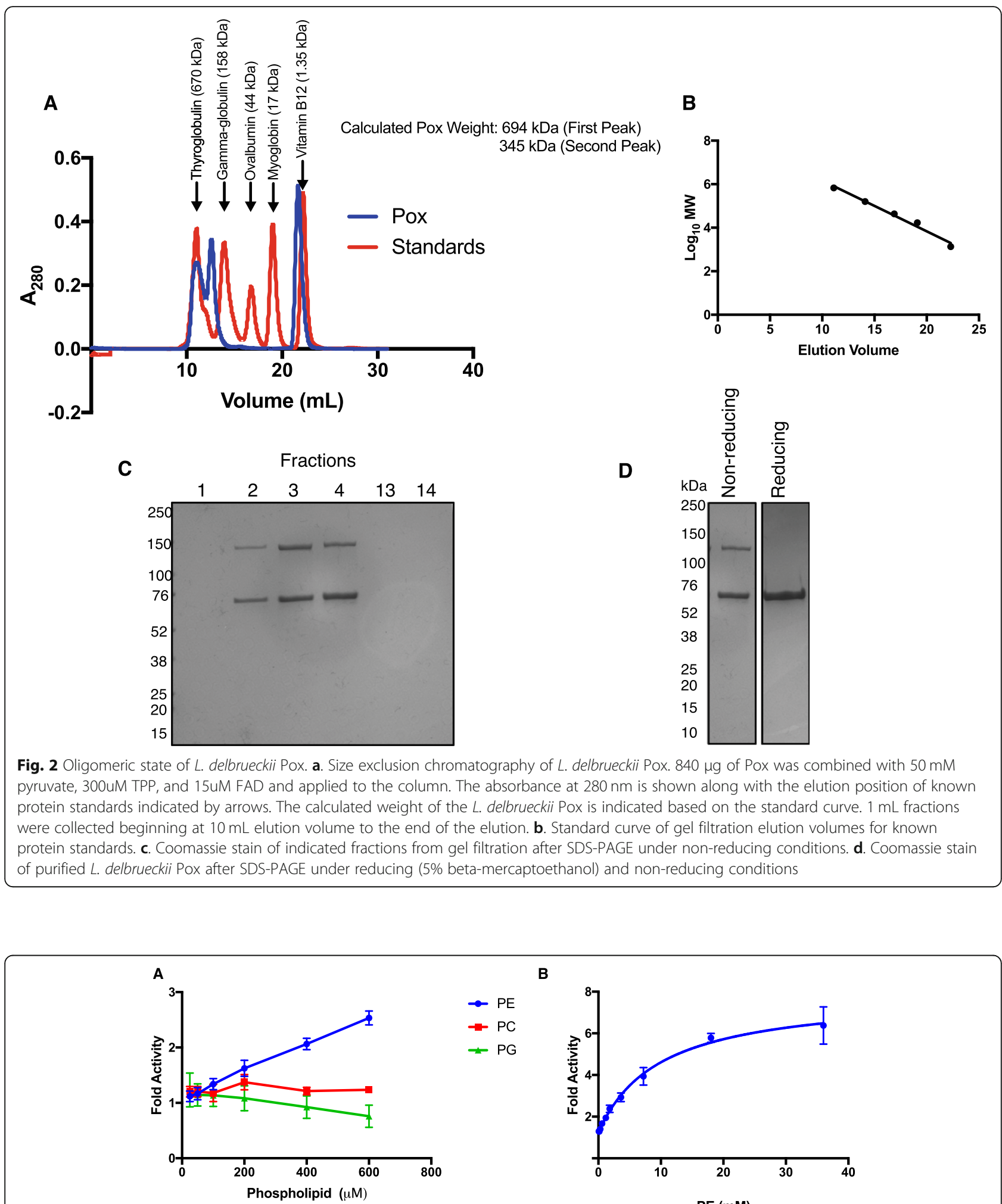

B
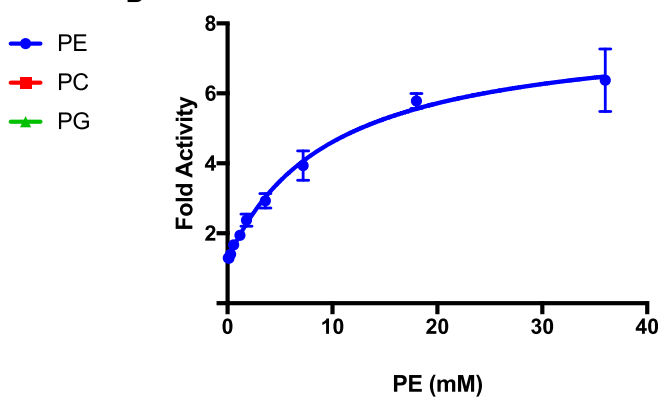

Fig. 3 L. delbrueckii Pox is activated specifically by phosphotidylethanolamine. a. Shown is the fold activity of L. delbrueckii Pox relative to the enzyme activity with no added phospholipid with various concentrations of phosphotidylethanolamine (16:0-18:1) (PE), phosphotidylcholine (16:0-18:1) (PC), and phosphotidylglycerol (16:0-18:1) (PG). Data represent the average of two independent experiments and error bars represent the standard error. $\mathbf{b}$. Shown is the fold activity of L. delbrueckii Pox with various concentrations of PE relative to enzyme activity with no PE. Data represent the average of at least two independent experiments and error bars represent the standard error 


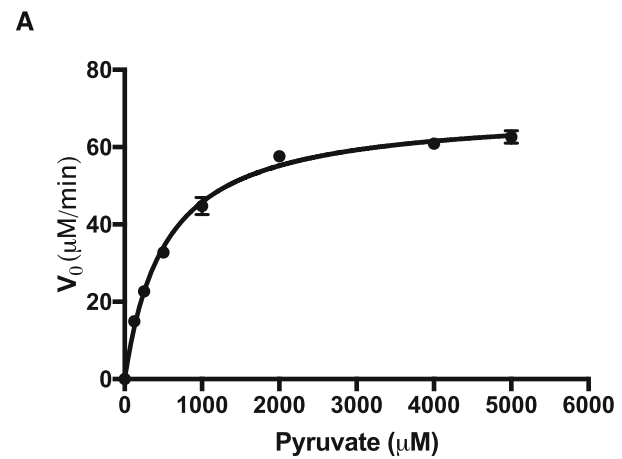

B

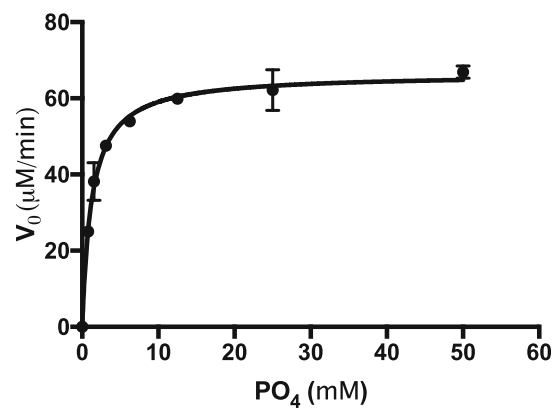

Fig. 4 Kinetic parameters of $L$. delbrueckii in the presence of PE. a. Shown is the enzyme velocity of $L$. delbrueckii Pox at various concentrations of pyruvate in the reaction mixture with $7.2 \mathrm{mM}$ PE. b. Shown is the enzyme velocity of $L$. delbrueckii Pox at various concentrations of phosphate in the reaction mixture with $7.2 \mathrm{mM}$ PE. In both panels, enzyme concentration was $170 \mathrm{nM}$. The data represent the average of two independent experiments and error bars represent standard error

consistent with the increase in $\mathrm{k}_{\text {cat }}$ observed in E. coli Pox and CidC upon phospholipid activation [3, 19]. The activation observed here is a hybrid of $\mathrm{V}$ - and $\mathrm{K}$-type activation since the $\mathrm{k}_{\text {cat }}$ increased and the phosphate $\mathrm{k}_{\mathrm{m}}$ decreased, which is also consistent with E. coli Pox $[9,19]$.

We also wanted to determine whether $L$. delbrueckii Pox inserts into PE micelles. We performed coprecipitation experiments with $L$. delbrueckii Pox in the presence and absence of PE. Nearly all of the enzyme remained in the soluble fraction and did not coprecipitate with $\mathrm{PE}$, which is in contrast to E. coli Pox [7] (Fig. 5).

\section{The C-terminus of L. delbrueckii pox is important for lipid activation}

The C-terminus of E. coli Pox is important for lipid activation of the enzyme [7]. To test if the C-terminus was important in the L. delbrueckii Pox, we generated a Cterminal truncation that lacked the last 34 amino acids. We purified the enzyme and tested it for its ability to be activated by PE (Fig. 6a). The enzyme had a specific activity of approximately $1.32 \mathrm{U} / \mathrm{mg}$ in the absence of PE, which is greater than the specific activity of the fulllength enzyme (Fig. 1c and Fig. 6b). This is consistent with the E. coli pox truncation having greater activity partially mimicking lipid activation [20]. Furthermore, the $\Delta 34$ enzyme was only weakly activated by PE to approximately 2 -fold greater than the enzyme without PE compared to an approximately 6 -fold activation with the full-length enzyme (Fig. 3b and Fig. 6c). This indicates

Table 3 Kinetic parameters of L. delbrueckii Pox with PE. Values For each parameter are listed with standard error

\begin{tabular}{lll}
\hline Km Pyruvate & Km PO4 & Kcat \\
\hline $519.9 \mu \mathrm{M} \pm 31.85$ & $1.25 \mathrm{mM} \pm 0.1336$ & $6.85 \mathrm{~s}-1 \pm 0.12$ \\
\hline
\end{tabular}

that the C-terminal sequence is important for the lipid activation of the Pox enzyme.

\section{Discussion}

Here, we demonstrated that the hydrogen peroxideproducing pyruvate oxidase enzyme from $L$. delbrueckii is catalytically activated specifically by PE, but not other phospholipids, and the last 34 amino acids of the enzyme are important for this activation. To our knowledge, this is the first hydrogenperoxide-producing Pox enzyme that has been shown to be activated by phospholipids. In addition, we showed that the $L$. delbrueckii Pox forms a pentameric structure in solution of which at least two subunits form a disulfide bond between cysteine 72. Other Pox enzymes form tetrameric structures, and there has been no evidence of disulfide bond formation indicating that the L. delbrueckii Pox has a different structure than other Pox enzymes [1, 4].

It is interesting that only PE had an impact on catalytic activity. This suggests that the size, shape, and charge of the phospholipid head group is important for catalytic activation. If the Pox enzyme is interacting with phospholipid that is already incorporated into the membrane, the head group would be exposed to the cytoplasm and be in

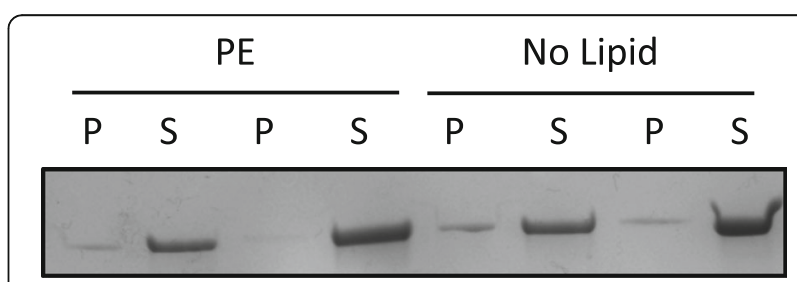

Fig. 5 L. delbrueckii does not co-precipitate with PE. L. delbrueckii Pox with $50 \mathrm{mM}$ pyruvate, 300uM TPP, and 15uM FAD was incubated with $1 \mathrm{mM}$ PE or no lipid. The lipid fraction was pelleted by centrifugation after $30 \mathrm{~min}$. Shown is a Coomassie stain of either the lipid pellet fraction (P) or the soluble fraction (S) 


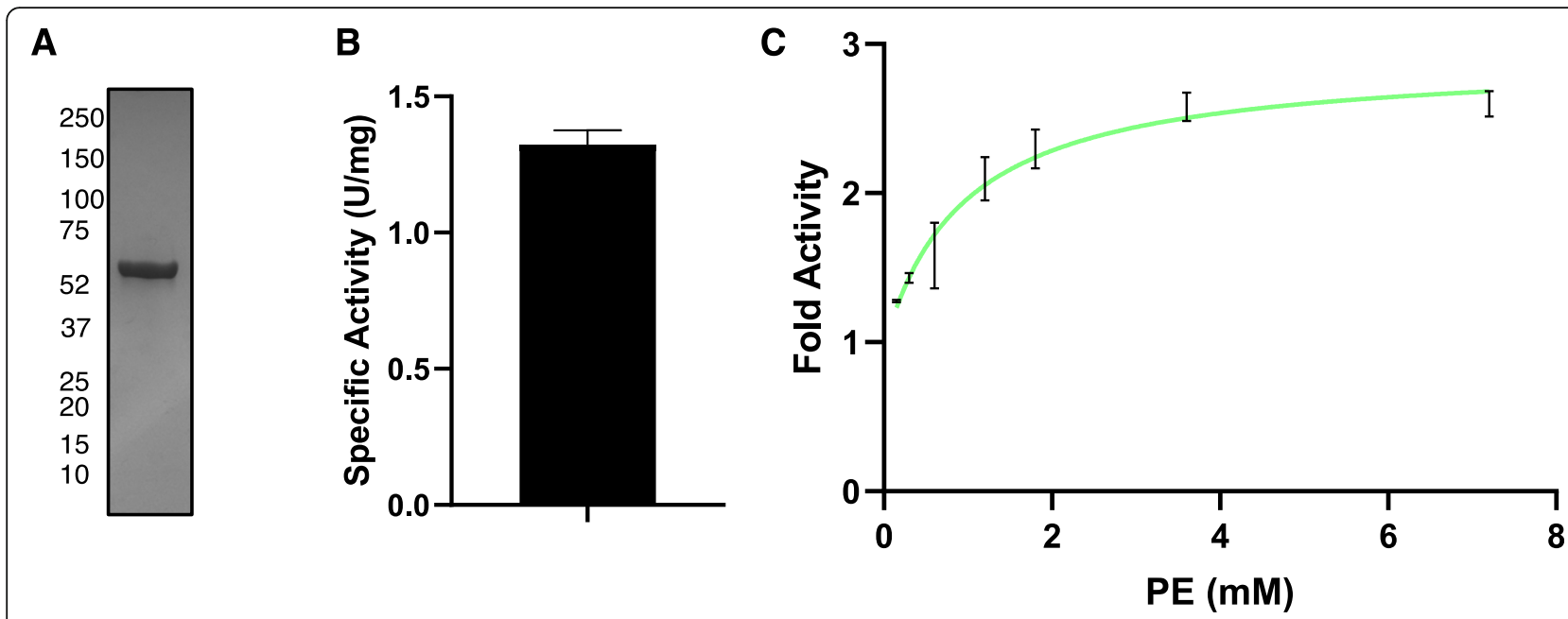

Fig. 6 The C-terminal 34 amino acids are important for lipid activation. a. Coomassie stain of purified $\Delta 34$ Pox enzyme. b. Specific activity of the purified $\Delta 34$ Pox enzyme. Data represent the average of two independent experiments and the error bar represent standard error. c. Shown is the fold activity of $\Delta 34$ Pox enzyme with various concentrations of PE relative to enzyme activity with no PE. Data represent the average of two independent experiments and error bars represent the standard error

the closest proximity to the enzyme. The interaction with the cytoplasm-facing ethanolamine likely occurs through the C-terminal 34 amino acids. Similar to the E. coli Pox, we hypothesize that this interaction induces a conformational shift in the enzyme that enhances electron transfer between FAD and TPP and decreases the phosphate $\mathrm{k}_{\mathrm{m}}$. This interaction is likely mediated by electrostatic forces. In fact, it is believed that CidC interaction with phospholipids is driven primarily by electrostatic forces suggesting there is interaction with the headgroup [3]. Furthermore, $\mathrm{PE}$ is known to be an abundant component of bacterial membranes, whereas $\mathrm{PC}$ is more commonly a eukaryotic and mammalian membrane component [21, 22]. Thus, activation only by $\mathrm{PE}$ is reasonable since $L$. delbrueckii Pox is a bacterial enzyme. Enhanced hydrogen peroxide production by $L$. delbrueckii could be beneficial in a complex microbial community. In fact, hydrogen peroxide production by Pox has been shown to be important in the inhibition of dental pathogens like $P$. gingivalis [16].

The pentameric structure of $L$. delbrueckii Pox is different from what has been observed with $E$. coli Pox and L. plantarum Pox $[1,4]$. Based on our gel filtration data, the $L$. delbrueckii also forms a decamer or a dimer of pentamers, but it is unclear which of these may be occurring. Interestingly, we also observed formation of dimers linked by a disulfide bond which are a component of the pentameric structure since they were present in fractions with an elution volume consistent with pentameric molecular weight. Therefore, the structure of the pentamer must be comprised of either three monomers and one dimer or one monomer and two dimers.

E. coli Pox lipid activation is a mixture of $\mathrm{K}$ - and Vtype allosteric activation where the pyruvate $K_{m}$ decreases and the $\mathrm{k}_{\text {cat }}$ increases [19, 23]. The lipid activation observed with $L$. delbrueckii Pox was also a mixture of $\mathrm{K}$ - and V-type allosteric activation, however, the specific parameters that changed were different. The pyruvate $k_{m}$ increased slightly while the phosphate $k_{m}$ decreased. The $\mathrm{K}_{\mathrm{cat}}$ did increase substantially (18-fold) similar to the E. coli Pox. Since the changes in pyruvate and phosphate $\mathrm{k}_{\mathrm{m}}$ could offset one another, we believe that the lipid activation of $L$. delbrueckii Pox is primarily driven by increasing the enzyme turnover rate. This is consistent with structural data of $E$. coli Pox demonstrating that lipid activation's primary effect on the enzyme is to shift a phenylalanine closer to the interface between TPP and FAD to increase the efficiency of electron transfer [9]. This is thought to increase the enzyme turnover rate substantially. It is also in agreement with the activation of CidC where the activation of the enzyme was exclusively driven by a 10 -fold increase in $\mathrm{k}_{\text {cat }}$ [3].

E. coli Pox, CidC, and PQO are the only known lipidactivated Pox enzymes [3, 5, 7]. These Pox enzymes likely evolved this ability so that they are most active near or at the membrane where it can readily transfer electrons to a membrane electron shuttle [3, 9]. For this reason, E. coli Pox inserts and co-precipitates with lipids in the presence of substrate [7]. Likewise, the CidC pyruvate oxidase from $S$. aureus interacts with phospholipids, but the interaction is believed to be mediated by electrostatic forces indicating that there does not necessarily need to be insertion into the membrane [3]. We did not observe any co-precipitation of $L$. delbrueckii Pox with $\mathrm{PE}$ suggesting that there is no insertion or that the interaction may be unable to withstand centrifugal forces. The fact that L. delbrueckii Pox does not strongly 
associate with or insert into the membrane may be a mechanism to regulate hydrogen peroxide production. If the association were too strong or there was insertion into the membrane, excessive hydrogen peroxide production may intoxicate the cell.

L. delbrueckii Pox does not need to interact with the membrane since it does not transfer electrons to a membrane electron shuttle, but rather to oxygen. Therefore, the utility of lipid activation of $L$. delbrueckii Pox is less clear than it is for other pyruvate oxidase enzymes that transfer electrons to membrane-bound carriers. One hypothesis is that the lipid activation of a hydrogen peroxide-producing Pox could be beneficial because it would position hydrogen peroxide production at a peripheral point of the cell. Passive diffusion of hydrogen peroxide from this point would subject the interior of the cell to less hydrogen peroxide and excrete more through the membrane than if the enzyme were positioned in the middle of the cell. Thus, the lipid activation of the Pox enzyme in this case may reduce the self-toxicity of hydrogen peroxide production by $L$. delbrueckii by activating the enzyme only at the edges of the cell.

\section{Conclusions}

In this report, we describe the first hydrogen peroxideproducing Pox enzyme that is activated by the phospholipid phosphotidylethanolamine. We also demonstrated a different oligomeric structure than other known Pox enzymes. Together, these data demonstrate that Pox enzymes from different bacterial species can vary in their structure and function. As Pox is an important enzyme in mediating bacterial interactions and microbial community structure, enhancing pathogenicity, and for certain biosensor applications, further understanding of the biochemical characteristics of Pox are warranted.

\section{Methods}

\section{Pyruvate oxidase}

Pyruvate oxidase enzyme or pyruvate:oxygen 2oxidoreductase (phosphorylating) (EC 1.2.3.3) was derived from Lactobacillus delbrueckii STYM1 strain [16]. The pyruvate oxidase enzyme was purified as previously described [16].

\section{Purification of L. delbrueckii pox $\Delta \mathbf{3 4}$}

The L. delbrueckii pox deletion mutant was PCR amplified from STYM1 genomic DNA using the primers listed in Table 4. The PCR product was ligated into the pFLAGCTC vector (Sigma, St. Louis, MO) using the NdeI and XhoI (New England Biolabs, Ipswich, MA) restriction sites. The product was transformed into DH5 $\mathrm{E}$. coli and the construct was confirmed by PCR and sequencing. The construct was transformed into LOBSTR E. coli (Kerafast, Boston, MA), in which the $L$. delbrueckii $\Delta 34$ Pox was expressed as a C-terminal $\mathrm{His}_{6}$-Pox. A starter culture was diluted 1:100 into $40 \mathrm{~mL}$ of LB supplemented with $100 \mu \mathrm{g} /$ $\mathrm{mL}$ of carbenicillin (Sigma, St. Louis, MO) and grown at $37^{\circ} \mathrm{C}$ until the $\mathrm{OD}_{600}$ reached $0.4-0.8$. The cells were cooled on ice for $10 \mathrm{~min}$ and $1 \mathrm{mM}$ isopropyl- $\beta$-D-thiogalactopyranoside (IPTG) (Sigma, St. Louis, MO) was added and the cells incubated at $25^{\circ} \mathrm{C}$ with shaking for 20 h. The cells were harvested by centrifugation at $3200 \mathrm{x} \mathrm{g}$ for $20 \mathrm{~min}$ at $4{ }^{\circ} \mathrm{C}$ and resuspended in $1 \mathrm{~mL} 20 \mathrm{mM}$ BisTris $150 \mathrm{mM} \mathrm{NaCl} \mathrm{pH} 7$ with EDTA-free cOmplete protease inhibitor cocktail (Roche, Basel, Switzerland). The cells were lysed by sonication on ice and the soluble cell lysate was harvested by centrifugation at $16000 \mathrm{x}$ g for $15 \mathrm{~min}$ at $4{ }^{\circ} \mathrm{C}$. The soluble cell lysate was filtered through a $0.22 \mu \mathrm{m}$ polyvinylidene fluoride (PVDF) filter and applied to a 1 $\mathrm{mL}$ bed volume of HisPure Ni-NTA resin (Thermofisher, Waltham, MA). The column was washed twice with two column volumes of buffer containing $25 \mathrm{mM}$ imidazole (Fisher, Pittsburgh, PA) and the bound protein was eluted from the column in buffer containing $250 \mathrm{mM}$ imidazole. Protein purity was assessed by SDS-PAGE and Coomassie staining.

\section{Pyruvate oxidase activity assay and kinetic parameters}

Pyruvate oxidase activity was measured as previously described [16]. Briefly, $50 \mathrm{mM}$ sodium pyruvate, $15 \mu \mathrm{M}$ FAD (Sigma, St. Louis, MO), $300 \mu \mathrm{M}$ TPP (Sigma, St. Louis, MO), $0.03 \%$ N-ethyl-N-(2-hydroxy-3-sulfopropyl)-m-toluidine (Sigma, St. Louis, MO), 0.015\% 4aminoantipyrine (Sigma, St. Louis, MO), and $33 \mu \mathrm{g} / \mathrm{mL}$ horseradish peroxidase (Sigma, St. Louis, MO) were combined with purified pox enzyme. After a 10-min incubation at room temperature, sodium phosphate $\mathrm{pH} 5.6$ was added to $50 \mathrm{mM}$ final concentration to initiate the reaction. The formation of a quinoneimine dye at $25^{\circ} \mathrm{C}$ was measured by an increase in absorbance at $550 \mathrm{~nm}$ and the specific activity was calculated as described. 1 unit of Pox activity is defined as the production of $1 \mu \mathrm{mol}$ of hydrogen peroxide per minute and this was converted to $\mu \mathrm{M} / \mathrm{min}$ for kinetic analysis.

Table 4 Primers used in this study

\begin{tabular}{lll}
\hline Primer & Description or purpose & Sequence \\
\hline Pox_F_Ndel & Cloning of $\Delta 34$ pox into pFLAG-CTC & AGATATCATATGGCAAAAATTAAGGGCGCAAAC \\
Pox_R__34_Xhol & $\begin{array}{l}\text { Cloning of } \Delta 34 \text { pox into pFLAG-CTC, } \\
\text { includes His } s_{6} \text { tag and stop codon }\end{array}$ & AATTCCCTCGAGTTAGTGATGGTGATGGTGATGACTTCCTGCACCTGAAGCCGCGTCAATTTC \\
\hline
\end{tabular}


For $\mathrm{k}_{\mathrm{m}}$ determination of pyruvate and phosphate and determination of $\mathrm{k}_{\mathrm{cat}}$, dilutions of substrate were used instead of the $50 \mathrm{mM}$ concentration. Enzyme concentration was $1.76 \mu \mathrm{M} . \mathrm{V}_{0}$ for each substrate concentration was plotted and the data were fitted using Prism software (Graphpad, San Diego, CA) kcat non-linear regression function. For $k_{m}$ and $k_{c a t}$ determination in the presence of phosphotidylethanolamine (PE), PE was used at a concentration of $7.2 \mathrm{mM}$ and the enzyme concentration was $170 \mathrm{nM}$.

\section{Phospholipid activation assay}

Phosphotidylethanolamine (16:0-18:1) (Avanti Lipids, Alabaster, AL), Phosphotidylcholine (16:0-18:1) (Avanti Lipids, Alabaster, AL), and Phosphotidylglycerol (16:018:1) (Avanti Lipids, Alabaster, AL) dissolved in chloroform were purchased commercially at a concentration of $10 \mathrm{mg} / \mathrm{mL}$. The desired volume of $10 \mathrm{mg} / \mathrm{mL}$ phospholipid solution was evaporated under a steady stream of argon gas (Airgas, Radnor, PA) and dried in a vacuum desiccator for $1 \mathrm{~h}$ to evaporate residual chloroform and yield the desired weight of dried phospholipid. The phospholipids were resuspended in the appropriate volume of $20 \mathrm{mM}$ Bis-Tris $150 \mathrm{mM} \mathrm{NaCl} \mathrm{pH} 6$ to achieve the desired concentration and incubated at $37^{\circ} \mathrm{C}$ with shaking at 225 $\mathrm{rpm}$ for $30 \mathrm{~min}$. The suspension was vortexed for $30 \mathrm{~s}$ and vortexed immediately prior to each use.

Phospholipids were added to the pyruvate oxidase assay mixture at various concentrations and enzyme activity was measured as described above. Fold activity was calculated relative to enzyme activity without phospholipid.

\section{Gel filtration analysis}

Prior to analysis of the L. delbrueckii Pox, protein standards were mixed together and applied to the Superdex 200 column (GE healthcare, Chicago, IL) using a duoflow system (Biorad, Hercules, CA). The protein standards were thyroglobulin $(670 \mathrm{kDa})$, gamma-globulin $(150 \mathrm{kDa})$, ovalbumin $(44 \mathrm{kDa})$, myoglobulin $(17 \mathrm{kDa})$, and vitamin B12 $(1.35 \mathrm{kDa})$. Protein was eluted from the column using a mobile phase of $20 \mathrm{mM}$ Bis-Tris $\mathrm{pH}$ $6150 \mathrm{mM} \mathrm{NaCl}$ at $0.5 \mathrm{~mL} / \mathrm{min}$ flow rate. A standard curve was plotted using the peak elution volume for each standard versus the $\log _{10}$ of the molecular weight in daltons. Eight hundred forty $\mu \mathrm{g}$ of purified $L$. delbrueckii Pox was incubated in the presence of $50 \mathrm{mM}$ pyruvate, $300 \mu \mathrm{M}$ TPP, and $15 \mu \mathrm{M}$ FAD for $10 \mathrm{~min}$ at room temperature prior to application to the column. The absorbance at $280 \mathrm{~nm}$ was recorded over the elution. One $\mathrm{mL}$ fractions were collected after the void volume of $10 \mathrm{~mL}$ until the end of the elution. The peak volume for the Pox fractions was used to calculate the molecular weight according to the standard curve.
L. delbrueckii Pox in each fraction from gel filtration was visualized after non-reducing SDS-PAGE analysis with Coomassie staining. For reducing SDS-PAGE analysis, 5\% $\beta$-mercaptoethanol (Sigma, St. Louis, MO) was included in the sample buffer.

\section{PE co-sedimentation}

PE was prepared as described above for activation experiments. $3 \mu \mathrm{g}$ of $L$. delbrueckii Pox was incubated with 50 $\mathrm{mM}$ pyruvate, $300 \mu \mathrm{M}$ TPP, and $15 \mu \mathrm{M}$ FAD with either $1 \mathrm{mM} P E$ or no PE for $30 \mathrm{~min}$ at room temperature. The PE fraction was precipitated by centrifugation at 16,000 $\mathrm{x} \mathrm{g}$ for $20 \mathrm{~min}$ at $4{ }^{\circ} \mathrm{C}$. The supernatant was aspirated as the soluble fraction and the PE pellet was resuspened in an equal amount of buffer. The fractions were visualized by Coomassie staining after SDS-PAGE.

\section{Abbreviations \\ Pox: Pyruvate oxidase; FAD: Flavin adenine dinucleotide; TPP: Thiamine pyrophosphate; PQO: Pyruvate:quinone oxidoreductase; $\mathrm{K}$-type: $\mathrm{K}_{\mathrm{m}}$-driven; $\mathrm{V}$ - type: Velocity-driven; TCA: Tricarboxylic acid; PE: Phosphotidylethanolamine; PC: Phosphotidylcholine; PG: Phosphotidylglycerol; IPTG: Isopropyl-B-D- thiogalactopyranoside; PVDF: Polyvinylidene fluoride}

\section{Acknowledgments \\ Not applicable.}

\section{Authors' contributions}

LPC and LTH conceived of the experiments and study design. LPC performed and analyzed the experiments. LPC and LTH wrote and edited the manuscript. All authors have read and approved the manuscript.

\section{Funding}

This work was funded by the NIH/NIDCR R01 DE024308 (to L.T.H) and F31 DE025523 (to L.P.C). The funding agencies had no role in the design of the study, data collection, analysis, interpretation of data, or in writing the manuscript.

\section{Availability of data and materials}

All data generated or analyzed during this study are included in this published article. Raw data can be made available upon request from the corresponding author.

Ethics approval and consent to participate

Not applicable.

\section{Consent for publication \\ Not applicable.}

\section{Competing interests}

The authors declare that they have no competing interests.

Received: 13 November 2019 Accepted: 16 April 2020

Published online: 24 May 2020

\section{References}

1. Sedewitz B, Schleifer KH, Götz F. Purification and biochemical characterization of pyruvate oxidase from Lactobacillus plantarum. J Bacteriol. 1984;160(1):273-8.

2. Cunningham CC, Hager LP. Reactivation of the lipid-depleted pyruvate oxidase system from Escherichia coli with cell envelope neutral lipids. J Biol Chem. 1975;250(18):7139-46.

3. Zhang X, Bayles KW, Luca S. Staphylococcus aureus CidC is a pyruvate: Menaquinone Oxidoreductase. Biochemistry. 2017;56(36):4819-29. 
4. Wang AY, Chang YY, Cronan JE. Role of the tetrameric structure of Escherichia coli pyruvate oxidase in enzyme activation and lipid binding. Biol Chem. 1991;266(17):10959-66.

5. Schreiner ME, Eikmanns BJ. Pyruvate:Quinone Oxidoreductase from Corynebacterium glutamicum: purification and biochemical characterization. J Bacteriol. 2005;187(3):862-71.

6. Chang Y-Y, Cronan JE. An Escherichia coli mutant deficient in pyruvate oxidase activity due to altered phospholipid activation of the enzyme. Proc Natl Acad Sci. 1984;81(14):4348-52.

7. Grabau C, Chang YY, Cronan JE. Lipid binding by Escherichia coli pyruvate oxidase is disrupted by small alterations of the carboxyl-terminal region. J Biol Chem. 1989;264(21):12510-9.

8. Cunningham CC, Hager LP. Crystalline pyruvate oxidase from Escherichia coli III. PHOSPHOLIPID AS AN ALLOSTERIC EFFECTOR FOR THE ENZYME. J Biol Chem. 1971:246(6):1583-9.

9. Neumann P, Weidner A, Pech A, Stubbs MT, Tittmann K. Structural basis for membrane binding and catalytic activation of the peripheral membrane enzyme pyruvate oxidase from Escherichia coli. Proc Natl Acad Sci. 2008; 105(45):17390-5

10. Taniai H, lida K, Seki M, Saito M, Shiota S, Nakayama H, et al. Concerted action of lactate oxidase and pyruvate oxidase in aerobic growth of Streptococcus pneumoniae: role of lactate as an energy source. J Bacteriol. 2008;190(10):3572-9.

11. Muller YA, Schulz GE. Structure of the thiamine- and flavin-dependent enzyme pyruvate oxidase. Science. 1993;259(5097):965-7.

12. Ramos-Montañez S, Tsui H-CT, Wayne KJ, Morris JL, Peters LE, Zhang F, et al. Polymorphism and regulation of the spxB (pyruvate oxidase) virulence factor gene by a CBS-HotDog domain protein (SpxR) in serotype 2 Streptococcus pneumoniae. Mol Microbiol. 2008;67(4):729-46.

13. Lorquet F, Goffin P, Muscariello L, Baudry J-B, Ladero V, Sacco M, et al. Characterization and functional analysis of the poxB gene, which encodes pyruvate oxidase in Lactobacillus plantarum. J Bacteriol. 2004;186(12):374959.

14. Spellerberg B, Cundell DR, Sandros J, Pearce BJ, Idänpään-Heikkilä I, Rosenow C, et al. Pyruvate oxidase, as a determinant of virulence in Streptococcus pneumoniae. Mol Microbiol. 1996;19(4):803-13.

15. Kreth J, Zhang Y, Herzberg MC. Streptococcal antagonism in Oral biofilms: Streptococcus sanguinis and Streptococcus gordonii interference with Streptococcus mutans. J Bacteriol. 2008 Jul 1;190(13):4632-40.

16. Cornacchione LP, Klein BA, Duncan MJ, Hu LT. Interspecies inhibition of Porphyromonas gingivalis by yogurt-derived Lactobacillus delbrueckii require active pyruvate oxidase. Appl Environ Microbiol. 2019;85(18):0127119.

17. Grabau C, Cronan JE. In vivo function of Escherichia coli pyruvate oxidase specifically requires a functional lipid binding site. Biochemistry. 1986;25(13): 3748-51.

18. Risse B, Stempfer G, Rudolph R, Möllering H, Jaenicke R. Stability and reconstitution of pyruvate oxidase from lactobacillus plantarum: dissection of the stabilizing effects of coenzyme binding and subunit interaction. Protein Sci. 1992:1 (12):1699-709.

19. Bertagnolli BL, Hager LP. Activation of Escherichia coli pyruvate oxidase enhances the oxidation of hydroxyethylthiamin pyrophosphate. J Biol Chem. 1991;266(16):10168-73.

20. Recny MA, Grabau C, Cronan JE, Hager LP. Characterization of the alphapeptide released upon protease activation of pyruvate oxidase. J Biol Chem. 1985;260(26):14287-91

21. Vance JE. Phospholipid synthesis and transport in mammalian cells. Traffic. 2015;16(1):1-18.

22. Zhang Y-M, Rock CO. Membrane lipid homeostasis in bacteria. Nat Rev Microbiol. 2008;6(3):222-33.

23. Mather MW, Gennis RB. Kinetic studies of the lipid-activated pyruvate oxidase flavoprotein of Escherichia coli. J Biol Chem. 1985;260(30):16148-55.

\section{Publisher's Note}

Springer Nature remains neutral with regard to jurisdictional claims in published maps and institutional affiliations.

Ready to submit your research? Choose BMC and benefit from:

- fast, convenient online submission

- thorough peer review by experienced researchers in your field

- rapid publication on acceptance

- support for research data, including large and complex data types

- gold Open Access which fosters wider collaboration and increased citations

- maximum visibility for your research: over $100 \mathrm{M}$ website views per year

At BMC, research is always in progress.

Learn more biomedcentral.com/submissions 